\title{
Erratum for Kinesiology Review 8(3)
}

The issue title and the order and form of the guest editors' names for the special issue of Kinesiology Review 8(3) were printed incorrectly on the cover and at the top of the table of contents. These have been corrected online and are as follows:

\section{Contemporary Youth Sport: Critical Issues and Future Directions}

Guest Editors: Alan L. Smith and Daniel Gould 